\title{
GrÉGOIRE-ThOMAs MONIOT Existence de surfaces de Willmore qui ne sont pas minimales
}

Annales de la faculté des sciences de Toulouse $\sigma^{e}$ série, tome 11, $\mathrm{n}^{\circ} 3$ (2002), p. 425-434

<http://www.numdam.org/item?id=AFST_2002_6_11_3_425_0>

(C) Université Paul Sabatier, 2002, tous droits réservés.

L'accès aux archives de la revue «Annales de la faculté des sciences de Toulouse » (http://picard.ups-tlse.fr/ annales/) implique l'accord avec les conditions générales d'utilisation (http://www.numdam.org/conditions). Toute utilisation commerciale ou impression systématique est constitutive d'une infraction pénale. Toute copie ou impression de ce fichier doit contenir la présente mention de copyright.

\section{NumDam}

Article numérisé dans le cadre du programme

Numérisation de documents anciens mathématiques

http://www.numdam.org/ 


\title{
Existence de surfaces de Willmore qui ne sont pas minimales ${ }^{(*)}$
}

\author{
GRÉGOIRE-Thomas MoNIOT ${ }^{(1)}$
}

RÉSUMÉ. - En utilisant un théorème de Langer et Singer, Pinkall a construit dans $[\mathrm{Pi}]$ une infinité de tores plongés dans $\mathrm{S}^{3}$ qui sont des points critiques de la fonctionnelle de Willmore, mais qui ne proviennent pas de surfaces minimales. Nous donnons ici une démonstration plus géométrique de ce résultat sans utiliser le théorème de Langer et Singer. Les tores obtenus par Pinkall sont des images réciproques par l'application de Hopf de courbes de $\mathbf{S}^{2}$. Lorsqu'un tel tore est extrémal, des relations entre la courbure de son image et la courbure des cercles d'une famille particulière enveloppant cette image apparaissent. Nous verrons que les contraintes apportées par ces relations sont suffisantes pour déterminer la courbure de ces images.

Abstract. - Using a theorem of Langer and Singer, Pinkall constructed in $[\mathrm{Pi}]$ an infinite series of embedded tori in $\mathbf{S}^{3}$ which are critical points of the Willmore functionnal, but that do not stem from minimal surfaces. We give here a more geometrical proof of this result whithout using the Langer and Singer's theorem. The tori obtained by Pinkall are inverse images by the Hopf application of curves in $\mathbf{S}^{2}$. Extremality of such a torus leads to some relations between the curvature of its image and the curvature of the circles of a particular family enveloping those images. We shall see that the constraints given by those relations are sufficient to determine the curvature of those images.

(*) Reçu le 22 janvier 2002, accepté le 22 octobre 2002

(1) Laboratoire de Topologie, Département de Mathématiques, UMR 5584, Université de Bourgogne, 9, avenue Alain Savary, BP 47870, 21078 Dijon Cedex, France.

e-mail : gtmoniot@u-bourgogne.fr 


\section{Introduction}

Soit $S$ une surface plongée dans $\mathbf{S}^{3}$ et $H$ sa courbure moyenne, la fonctionnelle :

$$
W(S)=\int_{S}\left(1+H^{2}\right) d A
$$

est appelée fonctionnelle de Willmore. Parmi les surfaces de genre un, on peut se demander quelles sont celles qui réalisent le minimum de $W$. C'est l'objet de la conjecture formulée par Willmore dans [Wi] qui propose comme candidats le tore appelé tore de Hopf ainsi que toutes ses images par les difféormorphismes conformes de $\mathbf{S}^{3}$. On trouve déjà trace de cette conjecture dans Thomsen [Th].

Dans [Pi], Pinkall construit des tores qui sont des images réciproques de courbes simples fermées de $\mathbf{S}^{2}$ par la projection de Hopf de $\mathbf{S}^{3}$ sur $\mathbf{S}^{2}$. Il montre que de telles surfaces sont des points critiques de $W$ si et seulement si les courbes correspondantes sont extrémales pour la fonctionnelle :

$$
F(\gamma)=\int_{\gamma}\left(1+k^{2}\right) d t
$$

où $k$ est la courbure de la courbe $\gamma \operatorname{de} \mathbf{S}^{2}$ et $d t$ sa longueur d'arc. L'existence de tores extrémaux est donc conditionnée par l'existence de courbes critiques pour $F$. Langer et Singer, dans [La-Si], ont mené l'étude des points critiques de la fonctionnelle :

$$
\int_{\gamma}\left(\lambda+k^{2}\right) d t
$$

où $\lambda$ est un réel et $k$ est la courbure géodésique d'une courbe $\gamma$ d'une variété riemannienne; la courbe $\gamma$ est, de plus, supposée fermée ou donnée avec une condition au bord. Ils ont nommé de telles courbes des courbes élastiques. S'appuyant sur cette étude, ils ont pu montrer qu'il existe une infinité de courbes fermées de $\mathbf{S}^{2}$ extrémales pour $F$. Ce dernier résultat permet alors à Pinkall de conclure à l'existence d'une infinité de tores plongés extrémaux.

On se propose ici d'adopter une autre démarche. En partant d'un tore $T$ plongé dans $\mathbf{S}^{3}$, on va considérer les sphères tangentes à ce tore de même courbure moyenne que celui-ci au point de tangence, sphères que l'on appellera sphères moyennes. Ces sphères forment une famille à deux paramètres qui possède une enveloppe à deux nappes dont l'une est le tore T. Bryant a montré qu'un tore est extrémal si et seulement si ses sphères moyennes sont aussi moyennes pour l'autre nappe. Or d'après Pinkall, une courbe de $\mathbf{S}^{2}$ est extrémale si et seulement si le tore qui est l'image inverse de cette courbe par la projection de Hopf est extrémal. On veut donc 
savoir s'il existe une condition du même ordre que celle de Bryant pour les courbes extrémales dans $\mathbf{S}^{2}$. Pour cela, on regardera ce que deviennent par la projection de Hopf, le tore $\mathrm{T}$, ses sphères moyennes et l'autre nappe. Les deux nappes sont envoyées sur deux courbes, et les sphères sur des disques tangents aux deux courbes. On verra de plus que dire que des sphères sont moyennes pour une des deux nappes équivaut à dire que les cercles qui sont les bords des disques ont pour courbure $\frac{1}{2}\left(k-\frac{1}{k}\right)$ où $k$ est la courbure de la courbe correspondante à la nappe choisie. On pourra alors conclure qu'une courbe $\gamma$ de courbure $k$ est extrémale si et seulement si les cercles tangents à $\gamma$ de courbure $\frac{1}{2}\left(k-\frac{1}{k}\right)$ enveloppent une autre courbe de même courbure que $\gamma$. Cette propriété sera suffisamment contraignante pour que l'on puisse en déduire rapidement l'équation différentielle satisfaite par la courbure de courbes extrémales. Cette équation différentielle aura pour solutions les solutions données par Langer et Singer, qui ont également montré qu'une infinité d'entre elles correspondent à des courbes fermées. Cela nous permettra alors de réaffirmer le résultat de Pinkall.

\section{Rappels et interprétations des résultats de Pinkall et Bryant}

Rappelons tout d'abord la description de l'application de Hopf choisie par Pinkall.

Il met en bijection $\mathbf{R}^{4}$ et $\mathbf{H}$ le corps des quaternions par l'application classique :

$$
\left(x_{1}, x_{2}, x_{3}, x_{4}\right) \longmapsto x_{1} \cdot 1+x_{2} \cdot i+x_{3} \cdot j+x_{4} \cdot k
$$

$\mathbf{S}^{3}$ est la sphère unité de $\mathbf{R}^{4}$ et $\mathbf{S}^{2}$ la sphère unité du sous-espace engendré $\operatorname{par} 1, j$ et $k$. Il définit la projection $\pi$ de Hopf par $\pi(q)=\tilde{q} q$ où $\tilde{q}$ est l'antiautomorphisme de $H$ qui fixe $1, j$ et $k$ et change $i$ en $-i$.

L'application notée $e^{i \phi}$ qui à un point $q$ de $\mathbf{S}^{3}$ associe le point $e^{i \phi} . q$ est telle que $\pi\left(e^{i \phi} . q\right)=q$. De plus, la fibre de Hopf contenant le point $q$ est l'ensemble $\left\{e^{i \phi} \cdot q\right\}_{\phi \in[0,2 \pi]}$.

Si $P$ est le sous-espace du plan tangent en $q$ à $\mathbf{S}^{3}$ de dimension deux perpendiculaire à la direction de la fibre de Hopf, alors l'application $d \pi$ restreinte à $P$ est une bijection qui double les distances.

Pinkall définit un tore de Hopf de la façon suivante. Soit $\gamma$ une courbe fermée simple dans $\mathbf{S}^{2}$. Le tore de Hopf $S$ associé à la courbe $\gamma$ est la surface $\pi^{-1}(\gamma)$. La courbure moyenne de ce tore en un point de $\pi^{-1}(\gamma(s))$ est égale à la courbure $k(s)$ de la courbe $\gamma$ au point $\gamma(s)$. On suppose que la courbe 
$\gamma(s)$ a pour paramètre sa longueur d'arc et que $L$ est sa longueur. De la précédente affirmation, Pinkall déduit :

$$
W(S)=\int_{\phi \in[0,2 \pi], t \in\left[0, \frac{L}{2}\right]}\left(1+k^{2}\right) d \phi d t=\pi \int_{s \in[0, L]}\left(1+k^{2}\right) d s=F(\gamma)
$$

ce qui montre que $S$ est extrémale pour la fonctionnelle de Willmore si et seulement si $\gamma$ est extrémale pour la fonctionnelle $F$.

Comme l'on veut travailler avec des familles de sphères, on peut étendre les calculs de Pinkall au cas d'une sphère $\mathcal{S}$ de $\mathbf{S}^{3}$ de courbure $b$. Pour cela, considérons la famille de sphères $\left\{e^{i \phi} . \mathcal{S}\right\}_{\phi \in[0,2 \pi]}$. Elle admet pour enveloppe un tore $T_{b}$ composé de fibres de Hopf. Cette surface $T_{b}$ se projette donc en une courbe $\gamma_{b}$ avec $T_{b}=\pi^{-1}\left(\gamma_{b}\right)$. La courbure de $\gamma_{b}$ est égale à la courbure moyenne de $T_{b}$. Or $T_{b}$ est de courbure moyenne constante égale à $\frac{1}{2}\left(b-\frac{1}{b}\right)$. La courbe $\gamma_{b}$ est alors un cercle de courbure $\frac{1}{2}\left(b-\frac{1}{b}\right)$. Elle forme le bord du disque en lequel se projette la sphère $\mathcal{S}$. De plus, si $\gamma$ est une courbe de $\mathbf{S}^{2}$, et si $\mathcal{S}$ est tangente à la surface $\pi^{-1}(\gamma)$, alors $T_{b}$ est tangente suivant toute la fibre de Hopf passant par le point de tangence entre $\mathcal{S}$ et $\pi^{-1}(\gamma)$ et par suite $\gamma_{b}$ est tangente à $\gamma$.

Lorsque $S$ est une surface dans $\mathbf{S}^{3}$, on peut considérer la famille des sphères, appelées sphères moyennes, qui lui sont tangentes et qui ont pour courbure la courbure moyenne de la surface au point de tangence considéré. Cette famille à deux paramètres de sphères admet une enveloppe à deux nappes. L'une est la surface $S$. Dans [Br], Bryant affirme qu'une condition nécessaire et suffisante pour que $S$ soit extrémale est que ces sphères soient aussi des sphères moyennes pour l'autre nappe.

Lorsque $\gamma$ est une courbe de courbure $k$, les sphères moyennes du tore de Hopf qui lui correspond ont pour courbure $k$ et se projettent en une famille de disques tangents à $\gamma$ dont le bord est un cercle de courbure $\frac{1}{2}\left(k-\frac{1}{k}\right)$.

On peut donc conclure :

LEMME 1. - Une courbe $\gamma$ de courbure $k$ est extrémale pour $F$ si et seulement si la famille de cercles tangents de courbure $\frac{1}{2}\left(k-\frac{1}{k}\right)$ admet une autre courbe enveloppe et si ces deux courbes ont même courbure aux points de contact d'un cercle avec chacune des deux enveloppes.

\section{Réduction du problème}

Supposons $\gamma$ extrémale. D'après le lemme 1 , il existe une courbe $\tilde{\gamma}$ qui a même courbure que $\gamma$. Il s'ensuit qu'il existe une isométrie de $\mathbf{S}^{2}$ qui envoie 
$\gamma$ sur $\tilde{\gamma}$. Le but de cette partie est de montrer qu'une étude approfondie n'est nécessaire que si cette isométrie est une symétrie par rapport à un grand cercle.

Il y a seulement trois types d'isométries pour $\mathbf{S}^{2}$ : les rotations, les symétries par rapport à un plan passant par l'origine que nous appellerons aussi symétries par rapport à un grand cercle, et les composés d'une rotation et d'une symétrie par rapport à un grand cercle invariant par la rotation. Notons $\mathcal{F}$ la famille de cercles considérés au lemme précédent.

Supposons que l'isométrie $I$ considérée soit la composée d'une rotation $R$ d'axe Nord-Sud et d'une symétrie $S$ par rapport au grand cercle $\mathcal{C}$ horizontal. Soit $p$ un point de $\gamma$ et $\tilde{p}$ le point de $\tilde{\gamma}$ qui lui correspond. On va montrer que la tangente à la courbe $\gamma$ ne peut avoir que deux directions possibles qui dépendent de $I$ et non de $\gamma$. Soit $\mathcal{C}_{1}$ et $\mathcal{C}_{2}$ les grands cercles passant par les deux pôles $N$ et $S$ et qui contiennent respectivement $p$ et $\tilde{p}$. L'angle $\alpha$ entre ces deux cercles est l'angle de la rotation. En opérant une projection stéréographique de pôle un point intersection de $\mathcal{C}$ avec le grand cercle passant par les deux pôles et faisant un angle $\alpha / 2$ avec $\mathcal{C}_{1}$ et $\mathcal{C}_{2}$, on obtient la situation représentée sur la figure 1 (a).

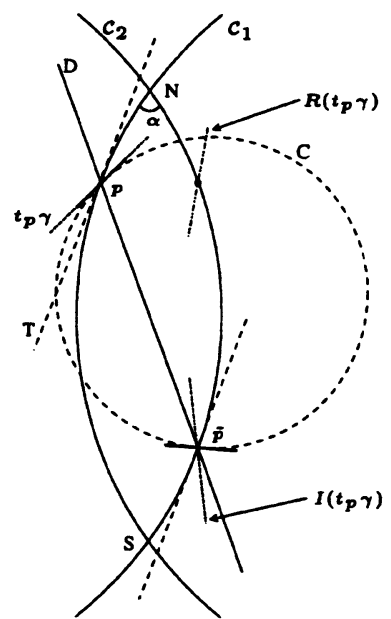

(a)

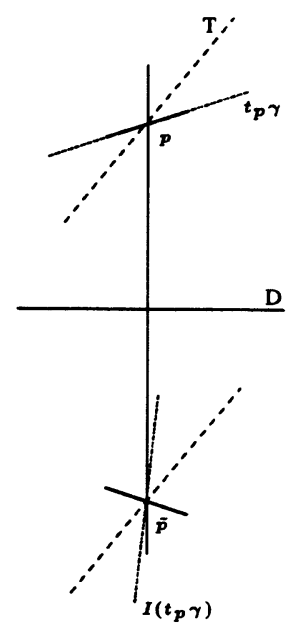

(b)

Figure 1

La tangente $t_{p} \gamma$ en $p$ à $\gamma$ est envoyée par l'isométrie sur la tangente $t_{\tilde{p}} \tilde{\gamma}$ en $\tilde{p}$ à $\tilde{\gamma}$. En outre, il existe un cercle $C$ de $\mathcal{F}$ qui est tangent à $\gamma$ en $p$. Ce cercle passe par $\tilde{p}$ et y a une tangente $t_{\tilde{p}} C$. Par définition, ce cercle doit être tangent à $\tilde{\gamma}$, et on doit donc avoir $t_{\tilde{p}} C=t_{\tilde{p}} \tilde{\gamma}$. 
Sur la figure, l'application qui envoie $t_{p} \gamma$ sur $t_{\tilde{p}} \tilde{\gamma}$ est la composée de la translation $t_{\vec{p} \tilde{p}}$ de vecteur $\overrightarrow{p \tilde{p}}$ et de la symétrie $s_{T}$ par rapport à la droite $T$ tangente à $\mathcal{C}_{1}$ en $p$, alors que l'application qui envoie $t_{p} C$ sur $t_{\tilde{p}} C$ est la symétrie $s_{D}$ par rapport à la droite $D$ médiatrice des points $p$ et $\tilde{p}$. Ces transformations sont représentées sur la figure 1 (b). On doit donc avoir $t_{\vec{p} \tilde{p}} \circ s_{T}\left(t_{p} \gamma\right)=s_{D}\left(t_{p} \gamma\right)$. En supposant $p$ et $\tilde{p}$ distincts, ce n'est possible que si les droites $T$ et $D$ sont parallèles. Cela implique soit que $p$ et $\tilde{p}$ sont sur $\mathcal{C}$, soit que les cercles $\mathcal{C}_{1}$ et $\mathcal{C}_{2}$ sont confondus et donc que la rotation $R$ est d'angle $\pi$, auquel cas $I$ est l'application antipodale.

Dans le premier cas, les courbes $\gamma$ et $\tilde{\gamma}$ sont sur un même grand cercle. Les cercles de la famille $\mathcal{F}$ sont tous confondus dans ce cercle. La relation de courbure du lemme 1 est contredite.

Dans le deuxième cas, les cercles de la famille $\mathcal{F}$ sont des grands cercles de courbure nulle. La courbe $\gamma$ est un cercle de courbure 1.

Supposons que l'isométrie considérée soit une rotation $R$ d'axe vertical. On montre dans le même esprit que les seules possibilités sont que $\gamma$ soit tangente ou perpendiculaire à un des cercles invariants par la rotation. Par suite $\gamma$ est soit un de ces cercles soit un des grands cercles perpendiculaires aux cercles invariants par la rotation. Dans le premier cas, les deux courbes sont confondues en un même cercle et $\mathcal{F}$ est réduite à ce cercle, et la relation entre les courbures est contredite. Dans le second cas, les deux courbes sont des grands cercles. Mais dans ce cas elles sont de courbure nulle et les cercles $\mathcal{F}$ sont réduits à des points. Les deux courbes sont confondues et la rotation est l'identité.

Supposons que l'isométrie considérée soit une symétrie par rapport à un grand cercle. Toujours de la même façon, on voit qu'un cercle passe par les points $p$ et $\tilde{p}=I(p)$ en étant tangent à $\gamma$ et $\tilde{\gamma}$ seulement s'il est centré le long du grand cercle de symétrie. La famille $\mathcal{F}$ est donc une famille de cercles centrés suivant un grand cercle.

\section{Reformulation de la condition nécessaire et suffisante}

On va désormais supposer que toute courbe extrémale est enveloppe de cercles centrés suivant un grand cercle. Cette restriction ainsi que la connaissance du rapport entre la courbure de ces cercles et la courbure de la courbe va nous permettre de mener des calculs afin d'établir une nouvelle condition nécessaire et suffisante de l'extrémalité de la courbe : 
LEMMe 2.- Une courbe $\gamma$ de $\mathbf{S}^{2}$ est extrémale si et seulement si sa fontion courbure $k$, lorsque le paramètre est la longueur d'arc de $\gamma$, satisfait à l'équation différentielle :

$$
k^{\prime \prime}=-k^{2} \frac{k+\frac{1}{k}}{2}
$$

Nous allons tout d'abord introduire l'espace des cercles. Il permet d'appréhender facilement les relations entre la courbure des cercles d'une famille enveloppe d'une courbe et la courbure de cette courbe.

On note $M$ le produit scalaire de Minkowski dans $R^{4}$. Soit $\Gamma$ la variété de de Sitter constituée des points de norme de Minkowski 1 , et $\mathcal{C}$ le cône de lumière constitué par les droites isotropes passant par l'origine. Le plan affine $P=e_{4}+V e c t\left(e_{1}, e_{2}, e_{3}\right)$ coupe $\mathcal{C}$ suivant une sphère $\mathcal{S}$ de dimension 2 . La métrique de Minkowski restreinte à cette sphère est une métrique euclidienne dont on la munit. $\mathcal{S}$ est ainsi isométrique à $\mathbf{S}^{2}$, un point $\left(x_{1}, x_{2}, x_{3}\right)$ de $\mathbf{S}^{2}$ correspondant au point $\left(x_{1}, x_{2}, x_{3}, 1\right)$ de $\mathcal{S}$. Tout cercle de $\mathcal{S}$ est la trace d'un plan de dimension trois de type $(+,+,-)$, c'est-à-dire d'un plan dont le vecteur normal est de norme positive. Inversement, tout plan de ce type trace un cercle sur $\mathcal{S}$. $\Gamma$ repère tous les cercles de $\mathcal{S}$, c'est l'espace des cercles.

Soit $\gamma$ une courbe dans $\mathbf{S}^{2}$, on note $(\gamma, 1)$ la courbe qui lui correspond dans $\mathcal{S}$. On peut considérer son vecteur tangent $\gamma^{\prime}$ et $n$ son vecteur normal dans le plan tangent à $\mathbf{S}^{2}$, vecteurs auxquels correspondent les vecteurs $\left(\gamma^{\prime}, 0\right)$ et $(n, 0)$ pour la courbe $(\gamma, 1)$ de $\mathcal{S}$. Tout cercle tangent à $\gamma$ se décrit dans l'espace des sphères par le vecteur $\sigma=(n, 0)+t .(\gamma, 1)$ où $t$ est un réel. En effet, les cercles tangents à $\gamma$ sont les traces des plans passant par le point $\gamma$ et contenant le vecteur $\gamma^{\prime}$. Ils ont donc pour vecteur normal $n+t . \gamma$ et leur équation est de la forme $\langle n+t . \gamma, x\rangle=t$. Ils s'étendent en un 3-plan vectoriel de $R^{4}$ d'équation $M((n+t . \gamma, 0)+t .(0,1), x)=0$ qui a bien $\sigma$ pour vecteur normal. De plus, on peut vérifier que $t$ est la courbure du cercle en question.

Une famille de cercles tangents à $\gamma$ et de courbure $\mathcal{K}$ est donc la courbe $\sigma=(n, 0)+\mathcal{K} .(\gamma, 1)$ dans l'espace des sphères.

Par définition, on a $n^{\prime}=-k \cdot \gamma^{\prime}$, où $k$ est la courbure de $\gamma$. On trouve alors : $\sigma^{\prime}=(\mathcal{K}-k) \cdot\left(\gamma^{\prime}, 0\right)+\mathcal{K}^{\prime} \cdot(\gamma, 1)$

On voit que $M(\sigma,(\gamma, 1))=0$ et $M\left(\sigma^{\prime},(\gamma, 1)\right)=0$. Ceci traduit que $\gamma$ est l'enveloppe de la famille de cercles $\sigma$.

En calculant $\sigma^{\prime \prime}$, on trouvera : $\left.M\left(\sigma^{\prime \prime},(\gamma, 1)\right)=(\mathcal{K}-k)<\gamma^{\prime \prime}, \gamma\right\rangle$ et lorsque le paramètre de $\gamma$ est la longueur d'arc, l'égalité devient $M\left(\sigma^{\prime \prime},(\gamma, 1)\right)$ $=k-\mathcal{K}$. 
Si $\gamma$ est extrémale, on a vu qu'elle est enveloppe de cercles $\sigma$ centrés sur un grand cercle. On peut supposer que ce grand cercle est l'intersection de $\mathbf{S}^{2}$ avec le plan de vecteur normal $e_{3}$. On va tout d'abord étudier les relations entre une famille $\sigma$ de cercles de courbure $\mathcal{K}$ centrés le long de ce grand cercle et $\gamma$, une de ces deux courbes enveloppes.

Pour repérer les cercles $\sigma$, on considère $\theta(s)$ l'angle du centre du cercle $\sigma(s)$ avec le vecteur $e_{1}$. Les cercles $\sigma(s)$ s'écrivent :

$$
\sigma(s)=\operatorname{ch}(l(s))\left(\cos (\theta(s)) e_{1}+\sin (\theta(s)) e_{2}\right)+\operatorname{sh}(l(s)) e_{4}
$$

où pour simplifier, on a introduit la fonction $l(s)$ qui est telle que $\operatorname{sh}(l(s))=$ $\mathcal{K}$. On a vu que $\gamma$ vérifie les équations suivantes :

$$
\begin{aligned}
M(\sigma,(\gamma, 1)) & =0 \\
M\left(\sigma^{\prime},(\gamma, 1)\right) & =0 \\
M\left(\sigma^{\prime \prime},(\gamma, 1)\right) & =(k-\mathcal{K})<\gamma^{\prime}, \gamma^{\prime}>
\end{aligned}
$$

Après avoir posé $A=<\gamma, \cos (\theta) e_{1}+\sin (\theta) e_{2}>$ et $B=<\gamma,-\sin (\theta) e_{1}+$ $\cos (\theta) e_{2}>$, on remplace $\sigma$ et ses dérivées par leur expression en fonction de $\theta$ et $l$ :

$$
\begin{aligned}
\operatorname{ch}(l) A-\operatorname{sh}(l) & =0 \\
l^{\prime} \operatorname{sh}(l) A+\theta^{\prime} \operatorname{ch}(l) B-l^{\prime} \operatorname{ch}(l) & =0 \\
\left(l^{\prime \prime} \operatorname{sh}(l)+\left(l^{\prime}\right)^{2} \operatorname{ch}(l)-\left(\theta^{\prime}\right)^{2} \operatorname{ch}(l)\right) A & \\
+\left(2 l^{\prime} \theta^{\prime} \operatorname{sh}(l)+\theta^{\prime \prime} \operatorname{ch}(l)\right) B-\left(l^{\prime \prime} \operatorname{ch}(l)+\left(l^{\prime}\right)^{2} \operatorname{sh}(l)\right) & =(k-\mathcal{K})<\gamma^{\prime}, \gamma^{\prime}>
\end{aligned}
$$

En éliminant $\mathrm{A}$ et $\mathrm{B}$, on obtient :

$$
\frac{\theta^{\prime \prime}}{\theta^{\prime}} l^{\prime}-\left(\theta^{\prime}\right)^{2} \operatorname{sh}(l) \operatorname{ch}(l)=l^{\prime \prime}-2\left(l^{\prime}\right)^{2} \frac{\operatorname{sh}(l)}{\operatorname{ch}(l)}+\operatorname{ch}(l)(k-\mathcal{K})<\gamma^{\prime}, \gamma^{\prime}>
$$

Supposons alors que $\gamma$ soit extrémale, on a $\mathcal{K}=\frac{1}{2}\left(k-\frac{1}{k}\right)$, c'est-à-dire $k=e^{l}$. On suppose de plus que $\gamma$ a pour paramètre sa longueur d'arc : $<\gamma^{\prime}, \gamma^{\prime}>=1$. Intéressons-nous à la valeur de $M\left(\sigma^{\prime}\right)$; d'une part $M\left(\sigma^{\prime}\right)=$ $(\mathcal{K}-k)^{2}=c h^{2}(l)$ et d'autre part $M\left(\sigma^{\prime}\right)=\left(\theta^{\prime}\right)^{2} c h^{2}(l)-\left(l^{\prime}\right)^{2}$. On obtient l'égalité supplémentaire :

$$
\left(\theta^{\prime}\right)^{2}=\frac{c h^{2}(l)+\left(l^{\prime}\right)^{2}}{c h^{2}(l)}
$$

Ce qui nous permet de connaitre aussi la valeur de $\theta^{\prime \prime} / \theta^{\prime}$. On déduit alors de (1) que

$$
l^{\prime \prime}+\left(l^{\prime}\right)^{2}=-e^{l} \operatorname{ch}(l)
$$


qui s'exprime à l'aide de $k$ comme :

$$
k^{\prime \prime}=-k^{2} \frac{k+\frac{1}{k}}{2}
$$

Réciproquement, prenons $\hat{\gamma}$ une courbe de $\mathbf{S}^{2}$ ayant pour paramètre sa longueur d'arc telle que sa courbure $\hat{k}$ vérifie l'équation différentielle (2). On définit alors une fonction $l$ telle que $e^{l}=\hat{k}$, et une fonction $\theta$ comme étant une primitive de $\sqrt{\frac{c h^{2}(l)+\left(l^{\prime}\right)^{2}}{c h^{2}(l)}}$.

On considère la famille des cercles $\sigma$ définie par les fonctions $l$ et $\theta$ que l'on vient de donner et $\gamma$ une des courbes enveloppes de cette famille. L'équation (1) donne dans ce cas :

$$
\operatorname{ch}(l)=(k-s h(l))<\gamma^{\prime}, \gamma^{\prime}>
$$

On a aussi les égalités $M\left(\sigma^{\prime}\right)=(k-s h(l))^{2}<\gamma^{\prime}, \gamma^{\prime}>$ et $M\left(\sigma^{\prime}\right)=$ $\left(\theta^{\prime}\right)^{2} \operatorname{ch}^{2}(l)-\left(l^{\prime}\right)^{2}$. D'où $(k-s h(l))^{2}<\gamma^{\prime}, \gamma^{\prime}>=\operatorname{ch}^{2}(l)$ et par suite $k-$ $\operatorname{sh}(l)=\operatorname{ch}(l)$ ce qui nous permet de conclure que $k=e^{l}=\hat{k}$ et aussi que $<\gamma^{\prime}, \gamma^{\prime}>=1$.

Les courbes $\gamma$ et $\hat{\gamma}$ ont alors toutes deux comme paramètre leur longueur d'arc et ont même courbure. Il existe donc une isométrie I de $\mathbf{S}^{2}$ qui envoie l'une sur l'autre. La famille de cercles $\sigma$ a deux courbes enveloppes symétriques par rapport à un grand cercle et qui ont donc même courbure. On en conclut que l'image de cette famille de cercles par l'isométrie I est une famille de cercles tangents à $\hat{\gamma}$ qui vérifie les conditions du lemme $1 . \hat{\gamma}$ est donc extrémale. La démonstration du lemme 2 est alors achevée.

\section{Conclusion}

Nous rejoignons maintenant l'article de Langer et Singer. Ils proposent comme courbes solutions du problème, les courbes dont la fonction de courbure est de la forme :

$$
k(s)=k_{0} \operatorname{cn}\left(\frac{k_{0} s}{2 p}, p\right)
$$

où $k_{0}$ est une constante, $p$ vaut $k_{0} / \sqrt{2\left(1+{k_{0}}^{2}\right)}$ et cn est la fonction cosinus elliptique. On peut vérifier que cette fonction vérifie l'équation différentielle du lemme 2 .

Pour chaque valeur de $k_{0}$, il existe une infinité de courbes de $S^{2}$ toutes isométriques qui ont $k(s)$ comme courbure. A chacune de ces courbes est 
associé un grand cercle $C_{k_{0}}$ tel que celle-ci soit l'enveloppe de cercles centrés $\operatorname{sur} C_{k_{0}}$. On obtient donc une famille de courbes $\gamma_{k_{0}}$ sur $S^{2}$ toutes extrémales. Langer et Singer ont montré que pour tout $p$ et $q$ entiers tels que $\frac{p}{q}<1$, il existe $k_{0}$ tel que la courbe $\gamma_{k_{0}}$ coupe $C_{k_{0}}$ en des points qui font un angle de $\frac{p}{q} 2 \pi$. Il existe alors une infinité de $k_{0}$ pour lesquels la courbe $\gamma_{k_{0}}$ est une courbe simple fermée. C'est ce résultat qui permet à Pinkall d'affirmer l'existence d'une infinité de tores plongés qui sont des points critiques pour la fonctionnelle de Willmore.

\section{Bibliographie}

[Br] BRYANT (R.L.). - A Duality Theorem for Willmore Surfaces, Journal of differential geometry, 20, no. 1 (1984), p 23-53.

[Wi] Willmore (T.J.). - Note On Embedded Surfaces, An. st. Univ. Iasi, s.I.a. Mathematica, 11B (1965), 493-496.

[Pi] Pinkall (U.). - Hopf Tori in $\mathrm{S}^{3}$, Inventiones mathematicae, 81, no. 2 (1985), 379-386.

[La-Si] LANGer (J.) et Singer (D.A.). - Curve-straightening in Riemannian manifolds, Ann. Global Anal. Geom., 5, no. 2 (1987), 133-150.

[Th] Thomsen (G.). - Über Konforme Geometrie I : Grundlagen der Konformen Flächentheorie, Abh. Math. Sem. Hambourg, (1923), 31-56. 